assessment is likely to be added to the routine work up panel in the near future. ${ }^{4}$ Reflecting these guidelines, Rogers et al. describe that while chromosomal fragility assessment was performed in most children, telomere length assessment was only performed at diagnosis in one-third of them.

Registries are crucial tools in efforts to improve outcomes for patients with rare diseases and their families. They serve as a means of pooling rare data in a standardized format in order to achieve meaningful sample sizes for subsequent analysis and allow comparison to historical or international cohorts, facilitate collaboration, generate hypotheses for future testing, and provide a framework for annotated sample collection and translational research. Further, participation in registry reporting contributes to achieving consistent and complete work up of new cases and provides a means of formulation and distribution of educational opportunities including multidisciplinary discussions which are so often needed in the management of rare conditions. Registries allow for the identification of patients, informing epidemiology assessments and areas of need, and may assist with allocation of scarce resources. Registries may facilitate feasibility assessments of and planning for clinical trials. The importance of registries focused on AA in particular is reflected in the increasing number of publications describing national outcome data in AA.,12-14 In this edition of Haematologica, Rogers et al. have made an important contribution to this data pool, informing optimal diagnostic and therapeutic approaches and, equally importantly, highlighting opportunities for further research and discussion in pediatric AA.

\section{Funding}

LF is supported by a Higher Degree Fellowship in Bone Marrow Failure from Maddie Riewoldt's Vision.

\section{References}

1. Rogers ZR, Nakano TA, Olson TS, et al. Immunosuppressive therapy for pediatric aplastic anemia: a North American Pediatric Aplastic Anemia Consortium study. Haematologica. 2019;104(10):1974-1983.

2. Williams DA, Bennett C, Bertuch A, et al. Diagnosis and treatment of pediatric acquired aplastic anemia (AAA): an initial survey of the North American Pediatric Aplastic Anemia Consortium (NAPAAC). Pediatr Blood Cancer. 2014;61(5):869-874.

3. Camitta BM, Thomas ED, Nathan DG, et al. Severe aplastic anemia: a prospective study of the effect of early marrow transplantation on acute mortality. Blood. 1976;48(1):63-70

4. Killick SB, Bown N, Cavenagh J, et al. Guidelines for the diagnosis and management of adult aplastic anaemia. Br J Haematol. 2016;172(2):187-207

5. Dufour C, Pillon M, Passweg J, et al. Outcome of aplastic anemia in adolescence: a survey of the Severe Aplastic Anemia Working Party of the European Group for Blood and Marrow Transplantation. Haematologica. 2014;99(10):1574-1581.

6. Yoshida N, Kobayashi R, Yabe H, et al. First-line treatment for severe aplastic anemia in children: bone marrow transplantation from a matched family donor versus immunosuppressive therapy. Haematologica. 2014;99(12):1784-1791.

7. Bacigalupo A. How I treat acquired aplastic anemia. Blood. 2017;129(11):1428-1436.

8. Townsley DM, Scheinberg P, Winkler T, et al. Eltrombopag Added to Standard Immunosuppression for Aplastic Anemia. N Engl J Med. 2017;376(16):1540-1550.

9. Yoshizato T, Dumitriu B, Hosokawa K, et al. Somatic Mutations and Clonal Hematopoiesis in Aplastic Anemia. $N$ Engl J Med. 2015;373(1):35-47.

10. Lai TP, Wright WE, Shay JW. Comparison of telomere length measurement methods. Philos Trans $\mathrm{R}$ Soc Lond B Biol Sci. 2018;373(1741).

11. Ghemlas I, Li H, Zlateska B, et al. Improving diagnostic precision, care and syndrome definitions using comprehensive next-generation sequencing for the inherited bone marrow failure syndromes. J Med Genet. 2015;52(9):575-584.

12. Contejean A, Resche-Rigon M, Tamburini J, et al. Aplastic anemia in the elderly: a nationwide survey on behalf of the French Reference Center for Aplastic Anemia. Haematologica. 2019;104(2):256-262.

13. Vaht K, Goransson M, Carlson K, et al. Incidence and outcome of acquired aplastic anemia: real-world data from patients diagnosed in Sweden from 2000-2011. Haematologica. 2017;102(10):1683-1690.

14. Zhu XF, He HL, Wang SQ, et al. Current Treatment Patterns of Aplastic Anemia in China: A Prospective Cohort Registry Study. Acta Haematol. 2019:1-9.

\title{
Mastering the multitude of monocytoses
}

\section{Ulrich Germing and Norbert Gattermann}

\author{
Department of Hematology, Oncology and Clinical Immunology, Heinrich Heine University Düsseldorf, Düsseldorf, Germany \\ E-mail: ULRICH GERMING - germing@med.uni-duesseldorf.de
}

doi:10.3324/haematol.2019.227546

I $\mathrm{n}$ this issue of the journal, Valent and coworkers report on diagnostic criteria for chronic myelomonocytic leukemia (CMML), CMML variants, and pre-CMML conditions. ${ }^{1}$ These CMML disorders have always been like orphans, trying to find their place in a suitable environment. They are rare entities but share chromosomal, molecular, morphological, hematologic, clinical, and prognostic features with other diseases in the large family of myeloid malignancies. The French-American-British group classified CMML as a myelodysplastic syndrome (MDS), based on its similarity to refractory anemia with excess blasts, although CMML "may have little in common with MDS showing trilineage dyspoiesis" and despite the fact that CMML features only minimal dysplasia in the erythroid lineage.
Pathologists and hematologists felt uncomfortable since there are more differences than similarities between CMML and MDS. World Health Organization classifications placed CMML in a "hermaphrodite" position between myeloproliferative neoplasms (MPN) and $\mathrm{MDS},{ }^{3-5}$ taking into consideration that there are proliferative characteristics as well as hematopoietic insufficiency associated with some degree of myelodysplasia. Despite all these efforts, CMML was once described as "lost in classification", as none of the classifications adequately reflects the marked heterogeneity of this group of myeloid neoplasms. ${ }^{6}$

A major problem with all myeloid neoplasias presenting without significant excess of blasts, in particular the cases presenting with monocytosis, is demarcation from reactive 
changes. The assumption that this problem might easily be solved by detecting somatic mutations turned out to be a false hope. Matters were complicated by the discovery of "age-related clonal hematopoiesis", also known as "clonal hematopoiesis with indeterminate potential", and "clonal hematopoiesis with oncogenic potential". 9 These conditions are associated with well-known hemato-oncological driver mutations but do not necessarily lead to overt hematologic malignancy. The older the patient, the less certain we can be that detection of a somatic mutation is positive proof of malignant disease.

Against this background, an international working group of hematologists and hemotopathologists met in Vienna and developed proposals on how to tackle the diagnostic problems in the gray area between reactive monocytoses and acute leukemias with monocytosis. ${ }^{1}$

According to their suggestions, the heterogeneous group of reactive monocytoses is now complemented by "idiopathic monocytosis of undetermined significance", which is conceptually equivalent to idiopathic cytopenia of undetermined significance. The category of "idiopathic monocytosis of undetermined significance" includes patients with monocytosis that is neither attributable to a plausible medical cause nor identifiable as a clonal proliferation.

These reactive or unclear monocytoses should be differentiated from clonal disorders that carry a risk of progression to overt CMML or acute myeloid leukemia. Therefore, "clonal monocytosis of undetermined significance" was proposed, in order to classify a disorder that does not yet fulfill the formal criteria of CMML. Very recently, another group showed that patients with clonal monocytosis identified by targeted gene sequencing have a clinical outcome similar to that of those with overt World Health Organization-defined CMML. ${ }^{10}$ Accordingly, these "notyet-CMML" cases might also be called "clonal monocytosis of clinical significance". The relationship between these conditions and CMML resembles that between "clonal cytopenia of undetermined significance" and MDS. ${ }^{11}$ Other clonal entities between reactive monocytosis and CMML are "RASopathies", which can develop into juvenile myelomonocytic leukemia, as well as certain histiocytoses.

However, a clinical hematologist is more likely to encounter the three types of CMML, characterized by either a more dysplastic or a more proliferative appearance,

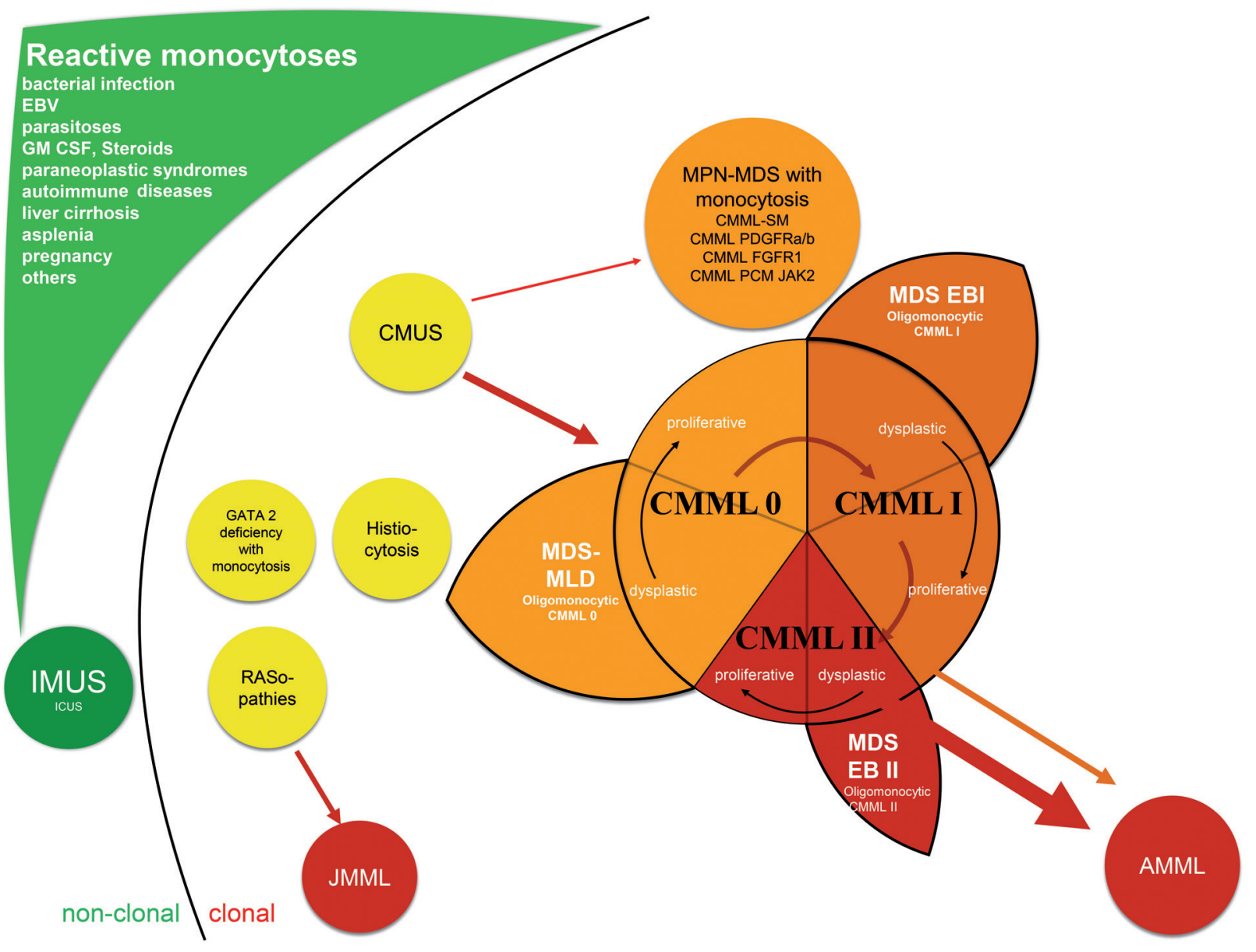

Figure 1. Relationship between different types of clonal monocytosis and demarcation from non-clonal, reactive monocytoses. EBV: Epstein-Barr virus; GM-CSF: granulocyte-macrophage colony-stimulating factor; IMUS: idiopathic monocytosis of undetermined significance; ICUS: idiopathic cytopenia of undetermined significance; CMUS: clonal monocytosis of undetermined significance; MDS-MLD: myelodysplastic syndrome with multilineage dysplasia; MPN-MDS: myeloproliferative neoplasm-myelodyplastic syndrome; CMML-SM: chronic myelomonocytic leukemia with systemic mastocytosis; MDS-EB: myelodysplastic syndrome with excess blasts; JMML: juvenile myelomonocytic leukemia; AMML: acute myelomonocytic leukemia. 
based on: (i) the percentage of bone marrow blasts, including promonocytes, and (ii) the white blood cell count in the circulation. The latter criterion, though, is artificial, since most CMML cases present with a white blood cell count of $8-14 \times 10^{9} / \mathrm{L}$, often oscillating around the cut-off value of $13 \times 10^{9} / \mathrm{L}$

All types of CMML show similarities with certain types of MDS. The paper by Valent et al. refers to a close relationship between MDS with multilineage dysplasia and dysplastic CMML-0, MDS with excess blasts-1 and dysplastic CMML-1, as well as MDS with excess blasts-2 and dysplastic CMML-2. In Figure 1, we try to illustrate the relationships within the large family of monocytoses.

Valent et al. propose the term "oligomonocytic CMML" in order to emphasize, as also pointed out by others, that it may be appropriate to diagnose CMML based on bone marrow monocytosis and CMML-typical somatic mutations, even if marked monocytosis is missing in the peripheral blood. ${ }^{12,13}$

The group also points out that CMML can display two different types of acceleration and progression. On the one hand, dysplastic CMML can adopt typical features of MPN by showing accelerated proliferation with organomegaly and constitutional symptoms, without necessarily producing an excess of blasts. This evolution from a dysplastic to a proliferative type may be heralded by increasing white cell counts, often accompanied by splenic enlargement. On the other hand, progression of CMML in terms of increasing blast percentage, i.e. from CMML-0 to CMML-1 or CMML-2, often occurs without a marked increase in white cell count or development of organomegaly. To complicate matters, both types of evolution can occur simultaneously or sequentially, and can be caused (or at least accompanied) by clonal evolution in terms of new somatic mutations, increased variant allele frequencies, or acquisition of chromosomal aberrations. As disease evolution may be more prominent in the bone marrow or peripheral blood, the authors recommend classifying the disease according to the highest blast count detectable.

Finally, the authors recommend complementing the group of CMML entities by including rare MPN-MDS variants, namely CMML with KITD816 $\mathrm{V}^{+}$systemic mastocytosis, and MDS-MPN with PDGFRA/B, FGFR1, or PCM-JAK2 rearrangements, because these rare entities are often associated with pronounced monocytosis.
The paper by Valent et al. provides a comprehensive description of all the diagnostic tools needed to assign patients to the appropriate category within the heterogeneous group of non-clonal and clonal monocytoses. It summarizes our current knowledge and represents a starting point for future refinements of the classification of bone marrow disorders characterized by monocytosis.

\section{References}

1. Valent P, Orazi A, Savona MR, et al. PProposed diagnostic criteria for classical chronic myelomonocytic leukemia (CMML), CMML variants and pre-CMML conditions. Haematologica. 2019;10410):1935-1949.

2. Bennett JM, Catovsky D, Daniel MT, et al. Proposals for the classification of the myelodysplastic syndromes. Br J Haematol. 1982;51(2):189199.

3. Brunning RD, Bennett JM, Flandrin G, et al. Myelodysplastic syndromes: introduction. In: Jaffe ES, Harris NL, Stein H, Vardiman JW editors. WHO Classification of Tumours of Haematopoietic and Lymphoid Tissues. 3rd ed. Lyon: IARC; 2001.

4. Orazi A, Bennett JM, Germing U, Brunning RD, Bain BJ, Thiele J. Chronic myelomonocytic leukemia. In: Swerdlow SH, Campo E, Harris NL, Jaffe ES, Pileri SA, Stein $\mathrm{H}$, et al., editors. WHO Classification of Tumours of Haematopoietic and Lymphoid Tissues. Lyon: IARC; 2008.

5. Orazi A, Bennett JM, Germing U, et al. Chronic myelomonocytic leukemia. In: Swerdlow SH, Campo E, Harris NL, Jaffe ES, Pileri SA, Stein $\mathrm{H}$, et al., editors. WHO Classification of Tumours of Haematopoietic and Lymphoid Tissues. Lyon: IARC; 2017.

6. Bowen DT. Chronic myelomonocytic leukemia: lost in classification? Hematol Oncol. 2005;23(1):26-33.

7. Jaiswal S, Fontanillas P, Flannick J, et al. Age-related clonal hematopoiesis associated with adverse outcomes. N Engl J Med. 2014;371(26):2488-2498.

8. Steensma DP, Bejar R, Jaiswal S, et al. Clonal hematopoiesis of indeterminate potential and its distinction from myelodysplastic syndromes. Blood. 2015;126(1):9-16.

9. Valent P, Kern W, Hoermann G, et al. Clonal hematopoiesis with oncogenic potential (CHOP): separation from CHIP and roads to AML. Int J Mol Sci. 2019;20(3).

10. Cargo C, Cullen M, Taylor J, et al. The use of targeted sequencing and flow cytometry to identify patients with a clinically significant monocytosis. Blood. 2019;133(12):1325-1334.

11. Cazzola M. Clonal monocytosis of clinical significance. Blood. 2019;133(12):1271-1272.

12. Geyer JT, Tam W, Liu YC, et al. Oligomonocytic chronic myelomonocytic leukemia (chronic myelomonocytic leukemia without absolute monocytosis) displays a similar clinicopathologic and mutational profile to classical chronic myelomonocytic leukemia. Mod Pathol. 2017;30(9):1213-1222.

13. Schuler E, Frank F, Hildebrandt B, et al. Myelodysplastic syndromes without peripheral monocytosis but with evidence of marrow monocytosis share clinical and molecular characteristics with CMML. Leuk Res. 2018;65:1-4.

\title{
p66Shc deficiency sets the scene for clinically aggressive chronic lymphocytic leukemia
}

\section{Richard Rosenquist}

\author{
Dept. of Molecular Medicine and Surgery, Karolinska Institutet, Stockholm, Sweden \\ E-mail: RICHARD ROSENQUIST - richard.rosenquist@ki.se
}

doi:10.3324/haematol.2019.225904

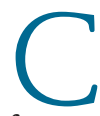
hronic lymphocytic leukemia (CLL) is a paradigmatic malignancy in which both cell-extrinsic (microenvironmental) and cell-intrinsic (genetic) factors contribute not only to the pathogenesis of the disease but also to disease evolution and outcome. ${ }^{1,2}$ In more recent years, the genomic landscape of CLL has been unraveled with the identification of "driver" gene muta- tions associated with clinical aggressiveness and chemorefractory disease, such as ATM, BIRC3, NOTCH1, NFKBIE, SF3B 1 and TP 53..$^{3-5}$ In addition to genetic aberrations, we also know that the $\mathrm{B}$-cell receptor (BCR) immunoglobulin plays a pivotal role in driving the disease onset and evolution. The somatic hypermutation status of IGHV genes divides patients into two major 